CHAPITRE 8

\title{
LE FRANCHISSEMENT DES BUSES, DES SEUILS EN ENROCHEMENTS ET DES OUVRAGES ESTUARIENS
}

\author{
M. LARINIER
}

CSP-CEMAGREF, GHAAPPE - Institut de Mécanique des Fluides

Avenue du Professeur Camille Soula - 31400 TOULOUSE

\section{LE FRANCHISSEMENT DES BUSES PAR LES POISSONS MIGRATEURS}

\subsection{Problèmes posés à la migration du poisson par les buses}

Les buses (circulaires, elliptiques, ovoïdes, buses-arches) et ponceaux (dalots, ouvrages voûtés, ponts-cadres), constituent une alternative intéressante - coûts de construction et de maintenance - aux ponts classiques pour le rétablissement des écoulements naturels lors des aménagements routiers ou autoroutiers.

En se substituant localement au milieu naturel, ces ouvrages modifient le substrat, les conditions d'éclairement et le régime d'écoulement des eaux.

Ils peuvent constituer des obstacles sérieux à la migration des poissons : vitesses trop fortes à l'intérieur de l'ouvrage (pouvant atteindre de $3 \mathrm{~m} / \mathrm{s}$ à $4 \mathrm{~m} / \mathrm{s}$ ), tirants d'eau insuffisants, chutes importantes à l'extrémité aval, enfin accumulation de débris représentent les causes les plus fréquentes de blocage pour les migrateurs.

Dès que la pente devient significative, l'écoulement subit une accélération brutale en tête de l'ouvrage. Compte tenu de la faible rugosité, les vitesses atteignent rapidement des valeurs importantes (l'écoulement pouvant même devenir torrentiel) et à peu près constantes tout au long de l'ouvrage : cette uniformité du champ des vitesses exclut toute zone de repos pour le poisson qui doit franchir la buse d'un seul trait. Lors des périodes de basses eaux, la profondeur d'écoulement devient très faible, insuffisante pour permettre la nage efficace du poisson.

Le blocage du poisson peut également se produire à l'extrémité aval de l'ouvrage lorsqu'il existe une chute, ce qui arrive fréquemment. Ce fait est quelquefois intentionnel, mais résulte le plus souvent d'un mauvais calage de la buse par rapport au profil du cours d'eau. II peut provenir aussi de l'abaissement du fil d'eau en aval, consécutif à l'installation de la buse si aucune mesure préventive (enrochements, seuils de contrôle) n'a été prise pour stabiliser le substrat.

Plus rarement, un mauvais calage de la tête de la buse peut se traduire par la création, dans le lit du cours d'eau en amont immédiat de l'ouvrage, d'une zone à fort gradient, infranchissable par le poisson.

\subsection{Lignes directrices pour la conception et l'installation de buses franchissables}

\subsubsection{Exigences pour le passage du poisson}

Dès que l'installation de la buse se traduit par la modification du régime hydraulique du cours d'eau, il convient de s'assurer que l'ouvrage ne fait pas obstacle au poisson.

Le fait qu'une buse soit franchissable ou non dépend des capacités de nage (en termes de vitesse de nage et d'endurance) des espèces présentes dans le cours d'eau et des conditions hydrauliques dans la buse en période de migration. 
Les capacités de nage sont généralement représentées par des courbes empiriques donnant la distance franchissable par un poisson de taille donnée dans un courant de vitesse donnée.

A l'aide de cette information, les conditions hydrauliques (vitesses moyennes à ne pas dépasser compte tenu de la longueur de l'ouvrage) permettant le passage du poisson peuvent être déterminées et prises en compte pour le dimensionnement de la buse.

Ces conditions doivent être satisfaites:

- pour l'individu de référence (espèce migratrice et taille) ayant les plus faibles capacités de nage susceptible d'emprunter la buse,

- pour l'ensemble des conditions de débit rencontrées en période de migration.

Il convient donc, comme pour tout dispositif de franchissement, de préciser le régime hydrologique du cours d'eau pendant la période de migration.

Si l'on possède la courbe des débits classés, on fera en sorte que la buse reste franchissable entre l'étiage et un débit qui n'est dépassé que $5 \%$ à $10 \%$ du temps dans l'année.

En l'absence de ces données, ce qui est le cas le plus fréquent sur les petits cours d'eau, on rendra la buse franchissable jusqu'à des débits voisins de 2 à 2.5 fois le module interannuel.

Outre certaines vitesses à ne pas dépasser, il conviendra de conserver une hauteur d'eau minimale dans la buse pour permettre le passage en étiage des plus gros individus, soit un minimum de $0.15 \mathrm{~m}$ pour la truite et de $0.30 \mathrm{~m}$ environ pour le saumon et la truite de mer.

\subsubsection{Critères de conception et d'installation d'une buse franchissable}

La forme de la buse détermine dans une certaine mesure les paramètres hydrauliques (hauteur d'eau, vitesse) qui conditionnent le passage du poisson. Les ouvrages à large fond (buses-arches, buses carrées, ponts-cadres) permettent de conserver une section de passage importante, relativement proche de celle du lit naturel. Ainsi, dans ce type d'ouvrage, à rugosité égale, la mise en vitesse sera moins importante que dans une buse circulaire. De plus, si la taille et les conditions d'installation de la buse (pente modérée, calage sous le lit naturel) le permettent, des graviers ou des galets, constituant une rugosité supplémentaire, pourront se déposer sur le fond et contribuer ainsi à réduire encore la vitesse de l'eau. Cependant, les buses circulaires et elliptiques s'avèrent à l'inverse préférables quand il s'agit d'assurer une profondeur d'eau minimale pendant la période d'étiage.

L'ouvrage est dimensionné pour évacuer des débits d'une durée de retour généralement importante (100 ans pour les autoroutes, de 25 à 100 ans pour les routes). La vitesse de l'eau dans la buse est calculée (formule de Manning-Strickler) pour le débit maximal considéré pour la période de migration en prenant en compte les caractéristiques de l'ouvrage (dimensions, forme de la section, rugosité, pente).

Considérant les critères de passage pour la ou les espèces envisagées, on détermine ainsi si le dimensionnement et le calage de l'ouvrage se révèlent compatibles avec le passage du poisson. remédier :

Si les critères de passage ne sont pas satisfaits, il existe plusieurs solutions pour y

- la meilleure solution consiste à substituer à la buse conventionnelle un ponceau ou une buse-arche dont les caractéristiques hydrauliques s'apparentent davantage à celles du cours d'eau naturel. On choisit un ouvrage d'une dimension suffisante pour maintenir une largeur et une section d'écoulement comparables à celles du cours d'eau pour les débits en période de migration. L'ouvrage est installé à la pente moyenne du cours d'eau sur le site, positionné au-dessous du lit du cours d'eau à l'amont comme à l'aval et rechargé avec des matériaux de la même granulométrie que ceux composant le lit. Cela 
revient en quelque sorte à reconstituer le lit du cours d'eau naturel à l'intérieur de l'ouvrage (KATOPODIS, 1984). II convient de vérifier que les matériaux composant la couche de surface sont suffisamment gros pour rester en place lors des crues. L'installation d'une buse franchissable par conception conduit généralement à un surdimensionnement de l'ouvrage par rapport aux stricts critères hydrauliques.

- on peut également réduire la vitesse dans l'ouvrage jusqu'au niveau adéquat en jouant sur la taille de la buse et/ou sur la pente du radier. Cette solution se traduit généralement, comme dans le cas précédent, par un surdimensionnement de la buse. II faut éviter cependant de caler l'ouvrage à une pente beaucoup plus faible que celle du cours d'eau, ce qui conduirait, soit à faire déboucher l'ouvrage au-dessus du lit naturel en aval (création d'une chute), soit à creuser le lit du cours d'eau en amont (création d'un tronçon à forte pente), conséquences également préjudiciables au passage du poisson,

- enfin, on peut installer à l'intérieur de l'ouvrage des rugosités artificielles, des déflecteurs, des plots, voire une passe à poissons classique.

Pour maintenir en permanence une profondeur d'eau minimale, en particulier en période d'étiage, le radier de la buse doit être placé à une trentaine de cm (WATTS, 1974 ; DRYDEN et STEIN, 1975 ; DANE, 1983) sous le niveau du lit naturel (Fig. 1).

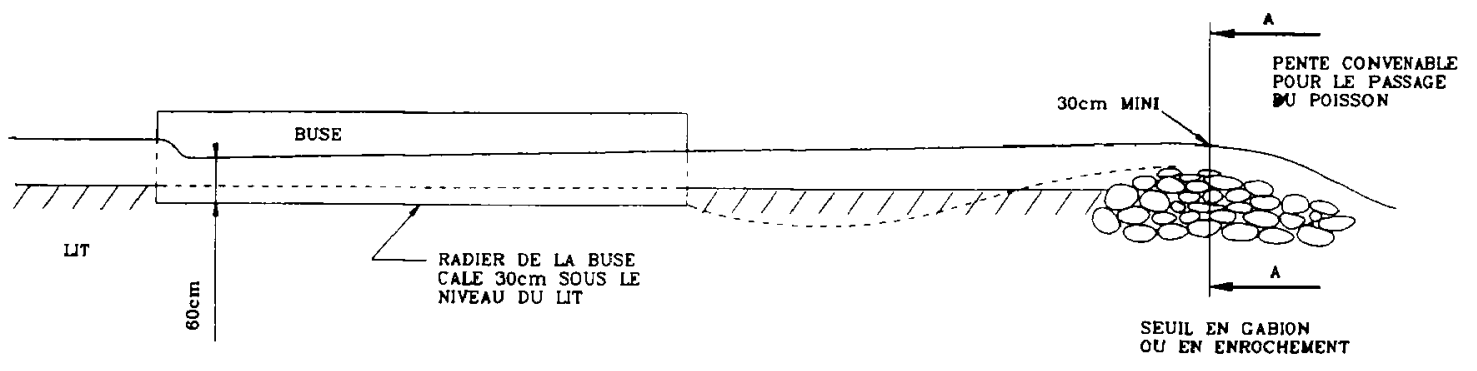

PROFIL EN LONG

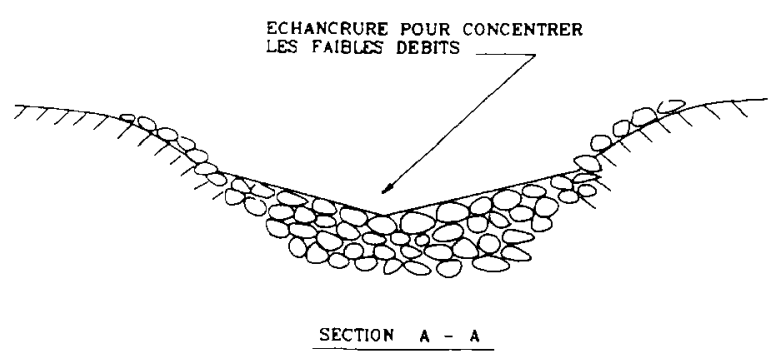

Figure 1 : Schéma illustrant le principe de l'implantation d'une buse avec noyage de son entrée par un seuil de contrôle en enrochements.

Figure 1 : View illustrating the installation of a culvert with a backwater rock sill.

Des protections de berges et un bassin de dissipation doivent être aménagés en aval de la buse. Ces aménagements sont destinés :

- à fournir aux poissons une zone de repos avant le passage dans la buse,

- à assurer un tirant d'eau minimum dans la partie aval de la buse (entrée du poisson) et à supprimer toute chute et accélération à l'entrée de la buse,

- à contrôler l'érosion en aval de la buse en dissipant l'énergie résiduelle de l'eau en sortie et à prévenir ainsi l'abaissement de la ligne d'eau.

Le bassin doit être d'un volume suffisant pour assurer une dissipation correcte de l'énergie sans turbulence ni aération excessives pour le poisson. II peut être construit en 
béton ou mieux, en enrochement, solution plus souple qui s'adapte aux évolutions éventuelles du lit.

Il convient de prévoir à l'aval de ce bassin un seuil de contrôle calé à une cote telle qu'il maintienne par noyage le niveau d'eau à une trentaine de centimètres au moins audessus du radier de la buse dans sa partie terminale afin d'éviter une accélération ou une chute. Dans certains cas, par mesure préventive ou pour remédier à une chute trop importante à l'extrémité aval, il peut s'avérer désirable, voire nécessaire, de construire plusieurs seuils de contrôle (prébarrages) sous l'ouvrage. Leur nombre dépend de la topographie. On divise la chute infranchissable en plusieurs petites chutes négociables par le poisson. Le dispositif se comporte comme une passe à poissons auquel on peut appliquer les critères de conception classiques; notamment la dénivellation entre seuils ne doit pas dépasser $0.25 \mathrm{~m}$ à $0.30 \mathrm{~m}$.

Tous ces seuils constituant des prébarrages doivent, par ailleurs, être pourvus d'échancrures pour concentrer les faibles débits et permettre ainsi le passage des poissons en période d'étiage.

\subsection{Déflecteurs}

L'expérience acquise en Amérique du Nord montre qu'il est de loin préférable de rendre la buse franchissable "par conception" plutôt que d'installer des dispositifs spéciaux (seuils, plots, déflecteurs) qui sont susceptibles d'induire des problèmes d'entretien sérieux et de réduire notablement, mais dans une proportion difficilement appréciable, les capacités d'évacuation de l'ouvrage (ENGEL 1974, DANE, 1983). L'installation de tels dispositifs s'avère cependant souvent indispensable pour corriger les ouvrages existants sur lesquels la migration du poisson n'avait pas été prise en compte initialement. D'autre part, lorsque la pente de l'ouvrage devient importante, l'installation de rugosités artificielles ou de déflecteurs devient souvent nécessaire ; ils serviront également à limiter les vitesses dans l'ouvrage et à protéger le radier lors des grosses crues.

On décrira dans la suite les dispositifs les plus couramment utilisés et qui paraissent les plus intéressants, soit par leur simplicité d'installation, soit par leur efficacité.

\subsubsection{Cloisons déversantes à crêtes horizontales, à échancrures ou fentes (Fig. 2)}

On installe dans la buse ou le ponceau une succession de seuils occupant toute la largeur destinés à assurer un tirant d'eau suffisant en étiage et garantissant des vitesses acceptables pour des débits plus importants. Une échancrure centrale ou latérale (carrée, rectangulaire ou triangulaire), ou une fente allant jusqu'au radier, dimensionnée pour le débit d'étiage, facilite le passage du poisson en basses eaux (RAJARATNAM et KATOPODIS, 1989, 1990).

Ce dispositif est assimilable à une passe à bassins classique et il peut être dimensionné en utilisant les mêmes critères, en particulier sur les charges minimales à prendre sur les seuils en fonction des espèces migratrices considérées. Les précautions particulières à prendre sont les suivantes:

- on veillera à donner des hauteurs aux murets telles que les critères sur les profondeurs minimales (Tmin) (respectivement $15 \mathrm{~cm}$ et $30 \mathrm{~cm}$ pour la truite et le saumon) soient respectées en particulier dans la zone la moins profonde généralement située à l'aval immédiat des seuils,

- le tirant d'eau au pied de chaque chute doit être au minimum égal à 2 à 2.5 fois la chute entre bassins pour faciliter le passage du poisson,

- l'espacement des seuils doit être suffisant, (supérieur à $8 p$ dans la mesure du possible, $p$ étant la hauteur des seuils). Si les seuils sont trop rapprochés, il se forme rapidement dès que le débit augmente des zones de recirculation participant peu à l'écoulement et peu dissipatrices d'énergie, l'écoulement passe alors rapidement en écoulement "quasi-lisse" de surface et à forte vitesse ("quasi-smooth flow" - KNIGHT et MCDONALD, 1979),

- la puissance dissipée volumique doit demeurer à un niveau acceptable (voir critères sur les passes à bassins). 


\subsubsection{Cloisons déversantes à crête triangulaire (Fig. 2)}

Par rapport à la solution précédente, les seuils à crête triangulaire auront l'avantage de créer des conditions d'écoulement hétérogènes, le poisson pouvant choisir la zone de passage qui lui convient le mieux en fonction des conditions de débit. La pente des côtés du triangle est de l'ordre de $1 / 5$ à $1 / 7$. En étiage, l'écoulement occupera seulement la partie basse du triangle. $\mathrm{Si}$, dans ces conditions, la charge amont (charge minimale Hmin) audessus de la pointe du triangle se révèle insuffisante pour assurer le passage du poisson, on concentrera, comme dans le cas des seuils horizontaux, ce débit dans une échancrure qui pourra être rectangulaire. Des critères de dimensionnement identiques aux seuils horizontaux peuvent être adoptés pour déterminer l'espacement et les hauteurs des cloisons.
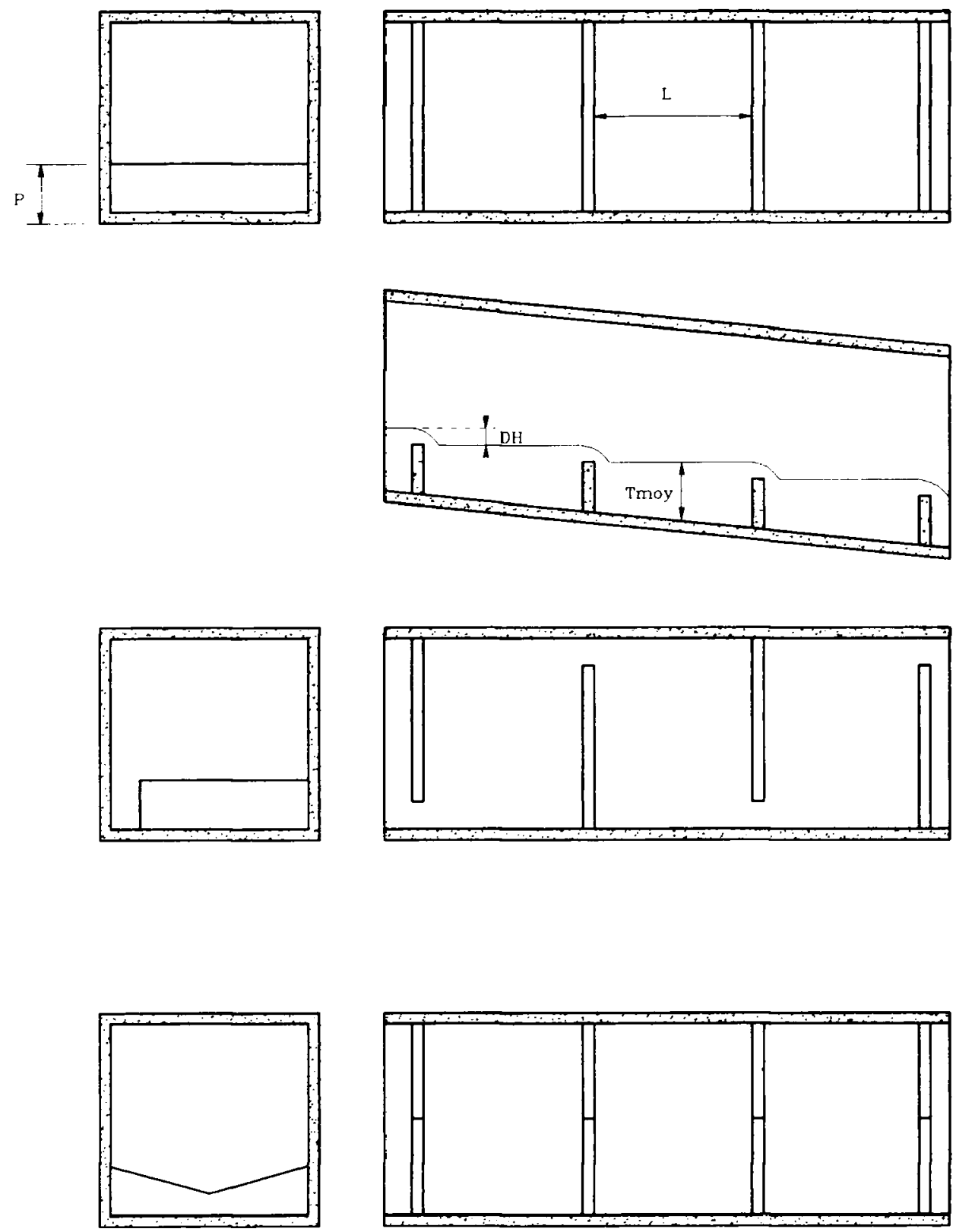

Figure 2 : Système de déflecteurs à seuils rectangulaires, avec fentes alternées et à seuils triangulaires.

Figure 2 : Rectangular, triangular and slotted-weir baffle systems. 


\subsubsection{Dispositifs “offset” (Fig. 3)}

Cette configuration de déflecteurs a été largement utilisée au Canada et aux USA (MCKINLEY et WEBB, 1966 ; ENGEL, 1974 ; RAJARATNAM et al., 1988) où elle a été adaptée à toutes les sections de buses. L'inconvénient majeur de ces dispositifs, satisfaisants d'un point de vue piscicole, est qu'ils sont plus délicats à installer et plus coûteux compte tenu de leur inclinaison, de leur espacement réduit et, par conséquent, de leur nombre.
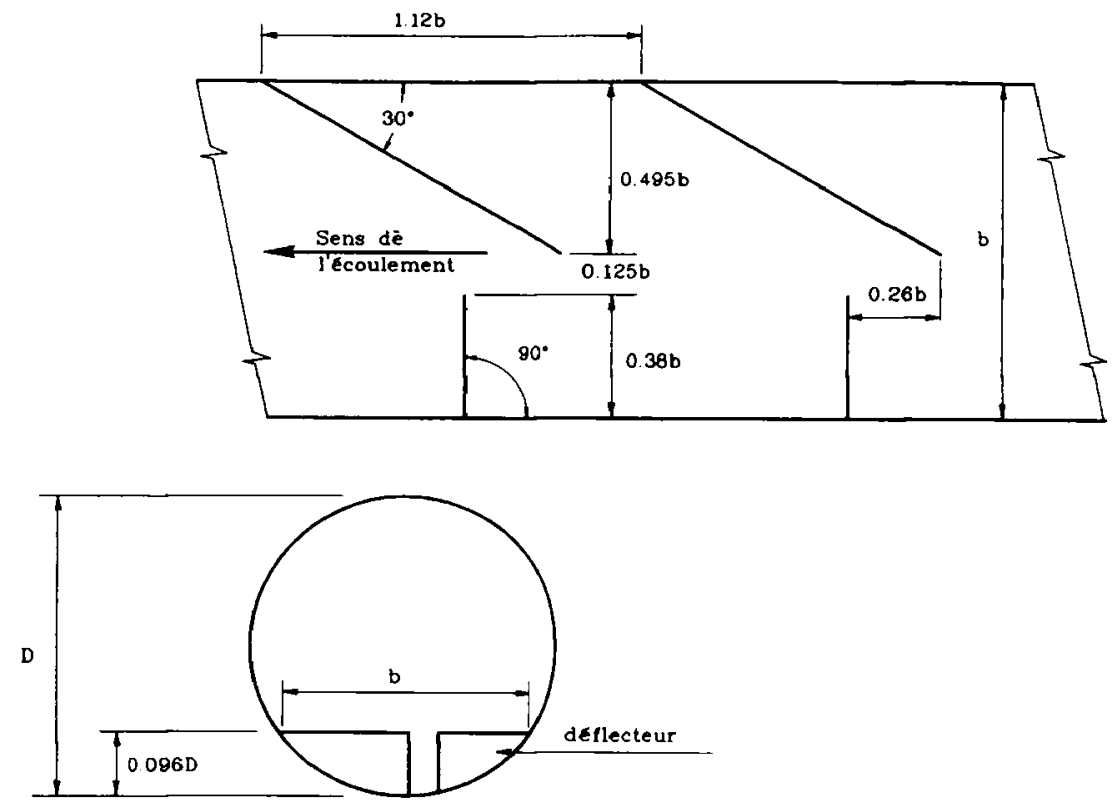

DISPOSITIF "OFFSET"

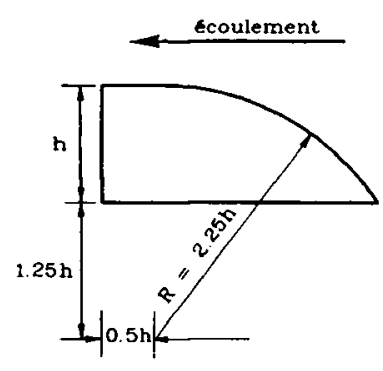

VUE LATERALE

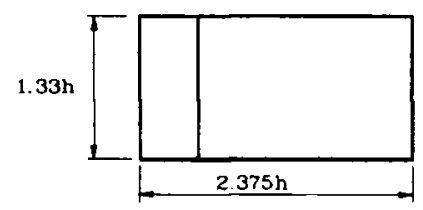

VUE DE DESSUS

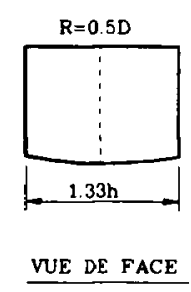

$\mathrm{h}=0.077 \mathrm{D}$

h mini $=30 \mathrm{~cm}$
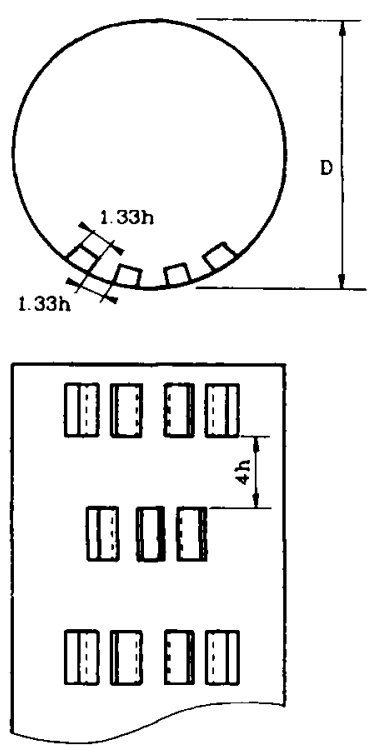

DISPOSITIF "SPOILER"

Figure 3 : Dimensions caractéristiques des déflecteurs de type "offset" et "spoiler". Figure 3 : Offset and spoiler baffle design dimensions. 
Les caractéristiques géométriques de ce système sont présentées sur la figure 3 , dimensions et espacements étant donnés sous forme adimensionnelle en fonction de la largeur du canal.

Les seuils ont une hauteur minimale de $0.30 \mathrm{~m}$. Pour les faibles débits, ce dispositif se comporte comme une passe à bassins à fentes verticales alors que pour les forts débits, l'orientation du muret latéral crée des courants hélicoïdaux, l'ouvrage se comportant alors comme une passe à ralentisseurs.

\subsubsection{Dispositif "spoiler" (Fig. 3)}

Ce système, étudié sur modèle réduit dans des buses circulaires (ENGEL, 1974 ; KATOPODIS et al., 1978), se compose d'une série de blocs profilés, régulièrement espacés et disposés en quinconce d'une rangée à l'autre. La forme de ces déflecteurs a été étudiée pour réduire au maximum leur impact sur l'hydraulique de la buse ainsi que les risques de colmatage par les débris tout en assurant au poisson, dans les zones de décollement, des aires de repos suffisantes. La hauteur minimale des "spoilers" recommandée est de 0.30 mètres.

II convient de vérifier que les vitesses des jets entre les "spoilers" restent compatibles avec les vitesses de nage des espèces concernées, la distance à franchir dans ces jets étant de l'ordre de 2.5 fois la hauteur des "spoilers". KATOPODIS (1978) donne la vitesse du jet sous forme adimensionnelle en fonction de la pente en intégrant les résultats obtenus par ENGEL (1974) sur modèle :

$$
\mathrm{V} /(\mathrm{gRh})^{0.5}=7.97 \text { 10.539. }^{0.5}
$$
la buse.

où $\mathrm{Rh}$ est le rayon hydraulique de la buse non équipée de déflecteurs et I la pente de

Testé sur des buses circulaires, le système peut néanmoins être adapté aux buses elliptiques ou aux buses-arches en ajoutant latéralement des blocs supplémentaires. ENGEL (1974) propose une expression permettant de calculer le nombre de blocs nécessaires sur la largeur:

$$
N=1 / 2(1+7 B / H)
$$

où $\mathrm{H}$ est la hauteur et $\mathrm{B}$ la largeur de l'ouvrage.

L'inconvénient majeur de ce dispositif est le nombre important de blocs, l'espacement latéral de deux blocs étant égal à leur largeur. II n'est d'autre part pas évident, en particulier dans le cas d'ouvrages elliptiques relativement larges, que le dispositif procure un tirant d'eau suffisant en étiage pour assurer le passage du poisson.

L'avantage des "spoilers" sur les autres dispositifs est qu'ils offrent moins de résistance à l'écoulement et sont moins sujets à l'engravement ou au colmatage par les corps dérivants.

\subsubsection{Impact des déflecteurs sur l'écoulement}

Tous les dispositifs précédemment évoqués ont été utilisés pour des pentes comprises entre $0.5 \%$ et $5 \%$ environ. Leur utilisation pour des pentes supérieures est à étudier avec soin.

Ils augmentent de façon notable le tirant d'eau dans l'ouvrage pour évacuer un débit donné, cette influence étant d'autant plus forte que le rapport tirant d'eau/hauteur des déflecteurs est important.

L'influence des seuils sur la capacité d'évacuation de l'ouvrage est liée à leur "rugosité", elle-même dépendante des rapports $L / p$ et $h / p$ (rapports de leur espacement et du tirant d'eau sur leur hauteur (SAYRE et ALBERTSON, 1961 ; KNIGHT et MCDONALD, 1979)).

Pour une hauteur de seuil de $0.30 \mathrm{~m}$ et un rapport $\mathrm{h} / \mathrm{p}$ de 6 , le coefficient de Strickler $(K)$ peut varier de 35 à 65 suivant l'espacement des seuils $(K=35,50,65$ pour $L p=7,5$ et 3.5). 
Pour un rapport tirant d'eau sur diamètre de buse $(h / D)$ compris entre 0.5 et 1 , le dispositif "offset" (de hauteur p/D égale à 0.10 ) réduit respectivement le débit d'un ordre de grandeur de $55 \%$ à $45 \%$ (RAJARATNAM et al., 1988).

\section{LE FRANCHISSEMENT DES SEUILS EN ENROCHEMENTS}

Les besoins croissants en matériaux de construction et de viabilité ont entraîné dans les années 1965-1975 un développement important des exploitations de granulats dans les lits mineurs des cours d'eau. Ces exploitations abusives entreprises souvent sous prétexte "d'entretien" du cours d'eau, ont induit de profondes nuisances : abaissement des lignes d'eau, déstabilisation des lits, érosion des berges, ruines des ouvrages de protection, déchaussement des ouvrages d'art, abaissement des nappes.

De très nombreux seuils en enrochements ont été installés pour tenter de redonner un nouveau profil d'équilibre aux cours d'eau et localiser les zones à fort gradient. La hauteur de ces seuils peut varier de moins de $1.50 \mathrm{~m}$ à plus de $8 \mathrm{~m}$.

Ils constituent le plus souvent des obstacles difficilement franchissables, sinon infranchissables, par les poissons migrateurs. Ces ouvrages sont le plus souvent installés perpendiculairement aux rives ; l'écoulement s'effectue de manière uniforme sur toute la largeur du seuil, la crête étant toujours horizontale. En eaux moyennes ou hautes, les vitesses sont trop importantes pour permettre le passage du poisson. La chute ne diminue généralement que très progressivement lorsque le débit augmente dans la mesure où les seuils pincent l'écoulement de façon à concentrer localement une dissipation d'énergie qui compense l'abaissement de la ligne d'eau. En étiage, l'eau percole à travers les enrochements, l'épaisseur insuffisante de la lame d'eau et sa vitesse rendant généralement difficile sinon impossible le passage des migrateurs.

Différentes solutions ont été envisagées pour rendre ces obstacles franchissables. Sur le Gave de Pau, certains seuils ont été équipés de passes à ralentisseurs utilisées également par les canoës-kayaks. Ce type de dispositif de franchissement s'est néanmoins révélé totalement inadéquat pour résoudre de façon satisfaisante ce genre de problème : il reste peu attractif compte tenu de la largeur des seuils et inadapté aussi bien aux espèces présentes sur le cours d'eau (de nombreux cyprinidés) qu'aux variations généralement notables du niveau d'eau amont.

Des passes à bassins à échancrure latérale et orifice noyé à gros débits (supérieurs à $1 \mathrm{~m}^{3} / \mathrm{s}$ ) constituent une meilleure solution, plus aftractive, mieux adaptée aux différentes espèces et aux fluctuations des niveaux. Elles sont cependant sujettes à l'engravement.

Sur l'Adour, plusieurs passes "rustiques" (chenal artificiel latéral à forte rugosité) à gros débits ont été installées en rive (seuil de Toulouzette). Elles nécessitent cependant, compte tenu de leur faible pente, un développement important qui rend leur implantation quelquefois difficile. Cette solution pourrait être adoptée chaque fois que possible pour l'aménagement des seuils existants.

La solution idéale (outre celle, évidente, consistant à ne plus tolérer de nouveau seuil) serait d'installer des seuils franchissables "par conception". Le principe serait :

— de réduire la hauteur de chute (à $1.0 \mathrm{~m}$ à $1.20 \mathrm{~m}$ au maximum),

- de donner à la crête un léger profil triangulaire (de l'ordre de 20 à $30 \mathrm{~cm}$ ) pour concentrer le débit dans la partie centrale en étiage et limiter la charge sur les bordures en eaux moyennes ou fortes,

- d'installer des "redans" dans les profils du seuil, de telle sorte que l'ouvrage se comporte comme une série de prébarrages.

Des essais ont été effectués de manière empirique sur certains seuils de l'Adour (Fig. 4). Un étude sur modèle réduit plus générale paraît indispensable.

Ces solutions se traduiraient par une augmentation des coûts de construction des seuils, mais éviteraient par contre l'installation d'un ouvrage en béton s'intégrant difficilement dans la structure du seuil et générant le plus souvent des problèmes d'entretien et de maintenance importants. 

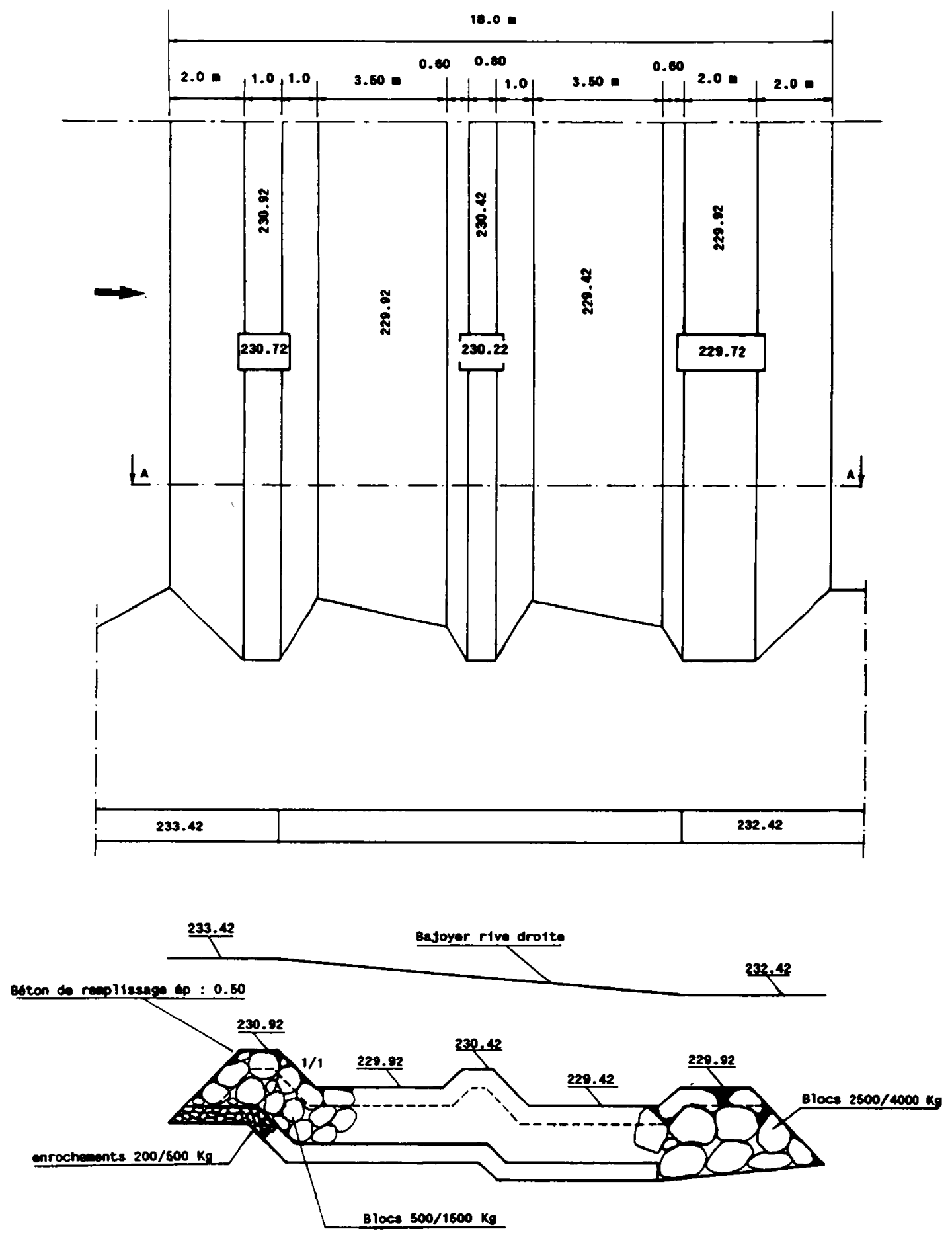

COPEA-S

Figure 4 : Seuils en enrochements en gradins sur l'Adour.

Figure 4 : Stepped rock sill on the Adour River. 


\section{LE FRANCHISSEMENT DES OBSTACLES ESTUARIENS ET COTIERS}

Certains aménagements particuliers constituent des obstacles à la migration plus par leur fonction que par leur hauteur. C'est le cas des clapets à marée ou des barrages estuariens destinés à interrompre l'écoulement de la marée dans la partie basse des estuaires afin de limiter les inondations, ou encore des aménagements destinés à créer des bassins à flot. Dans ce type d'obstacle, l'écoulement se fait de la rivière vers la mer à marée basse, puis dès que le niveau de la marée dépasse celui de la rivière, les clapets ou vannes se ferment, le niveau côté mer pouvant alors s'élever de plusieurs mètres audessus de celui de la rivière.

Deux facteurs viennent singulièrement compliquer le problème à résoudre :

- les niveaux de part et d'autre de l'obstacle sont éminemment variables : le niveau aval, compte tenu de la marée, et le niveau amont, suite au stockage de l'eau douce à marée haute. L'importance des fluctuations aval dépend de l'importance des marées (coefficient de marée) et de la distance de l'obstacle à la mer,

- la modification du gradient de salinité qu'induit la présence de l'obstacle : au lieu de bénéficier d'un gradient de salinité favorable à une adaptation progressive au changement de milieu, les migrateurs se trouvent confrontés à un changement brusque dont les effets biologiques (stress, mortalité) et comportementaux (retards ou blocage de la migration) sont encore mal connus.

De nombreuses rivières côtières de Seine-Maritime ont vu dès la fin du $19^{\mathrm{e}}$ siècle leur estuaire disparaître suite au busage de leur exutoire en mer. Elles transitent dans un canal couvert équipé généralement d'un clapet à marée destiné à éviter l'inondation à marée haute de l'ex-zone d'estuaire aujourd'hui viabilisée (EUZENAT et FOURNEL, 1983). Ces clapets sont constitués de un ou plusieurs épais panneaux de bois suspendus à une charnière horizontale.

Ces ouvrages constituent généralement un obstacle important à la migration des salmonidés : vitesses élevées dans les buses, mise en vitesse locale au niveau des clapets à marée basse, fermeture totale du clapet à marée haute interdisant le passage du poisson en amont.

Plusieurs solutions techniques ont été envisagées sur certains ouvrages particuliers. Les vitesses dans les buses peuvent être réduites en disposant des déflecteurs ou ralentisseurs sur le fond. Le passage au niveau des clapets peut être amélioré en ouvrant des "chatières" (ce qui a été fait sur certains ouvrages) d'une section suffisante ou en modifiant la conception des clapets.

Des essais sur modèle ont montré que le comportement des clapets (temps et degré d'ouverture) était très sensible à leur poids apparent, à la position de leur centre de gravité, au couple de rappel, etc... (I.M.F.T., 1988a, 1988b). Le passage des poissons peut être grandement facilité par le remplacement des clapets existants par des clapets plus légers et éventuellement des clapets monoblocs par des clapets à plusieurs éléments articulés.

Si les problèmes ont été identifiés et des solutions proposées, elles n'ont malheureusement pas encore été suivies de réalisation.

Récemment, il a été construit sur la Bresle, dans le port du Tréport, un bassin à flot comportant une écluse de navigation, un ouvrage de décharge constitué d'une vanneclapet ainsi qu'un dispositif de franchissement. Quel que soit l'état de la marée, le poisson peut parvenir à l'entrée de la passe grâce à une série de trois prébarrages garantissant un niveau d'eau minimal au pied de l'ouvrage. Le niveau amont est maintenu à la cote 7.5 , alors que le niveau aval - qui est celui de la mer - varie suivant l'état de la marée entre 4.20 et 10.00. Le dispositif de franchissement se compose de l'aval vers l'amont (Fig. 5) :

- d'une entrée basse, fonctionnant pour les niveaux aval compris entre 4.20 et 6.50 , équipée d'une vanne asservie,

- de deux volées d'une passe à ralentisseurs à chevrons épais (pente $16 \%$ ) amenant le poisson à la cote 6.75 , 
- d'une entrée haute, fonctionnant pour les niveaux aval supérieurs à 6.50 , équipée d'une vanne asservie,

— d'une section de passe à bassins permettant aux poissons de regagner l'amont.

Dès que le niveau d'eau côté mer atteint 6.50, l'entrée basse se ferme et l'entrée haute s'ouvre, permettant au poisson d'accéder directement dans la passe à bassins.

Le dispositif peut fonctionner en sens inverse (de la mer vers la rivière) comme passe à orifices noyés tant que le niveau de la marée reste inférieur à la cote 9.50 (EUZENAT et LARINIER, 1990 ; I.M.F.T., 1990).

Après une année de fonctionnement, aucun impact global de la construction du bassin à flot et de son dispositif de franchissement n'a pu être constaté au niveau de la station de contrôle des migrateurs située quelques kilomètres en amont.

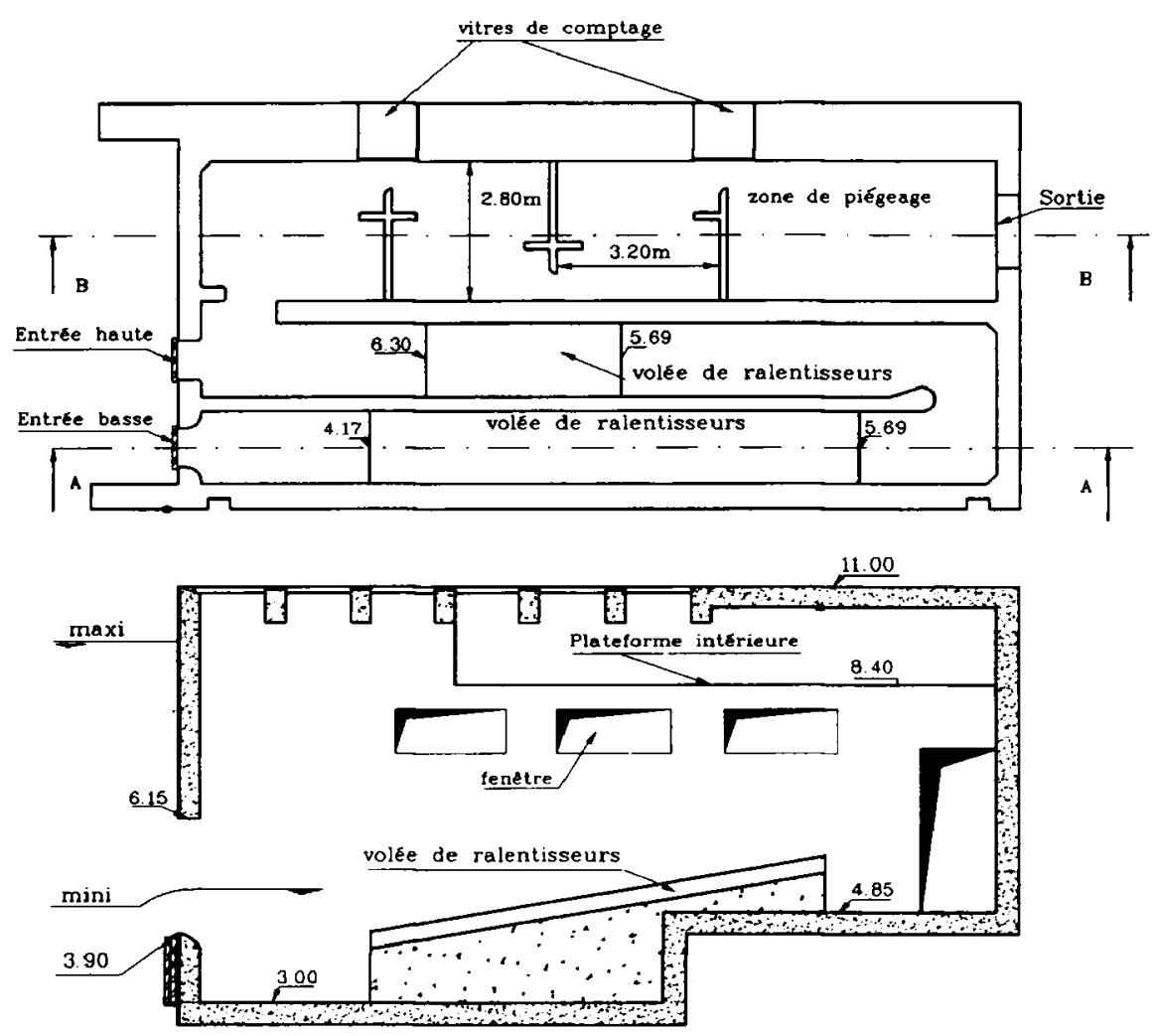

COUPE A-A

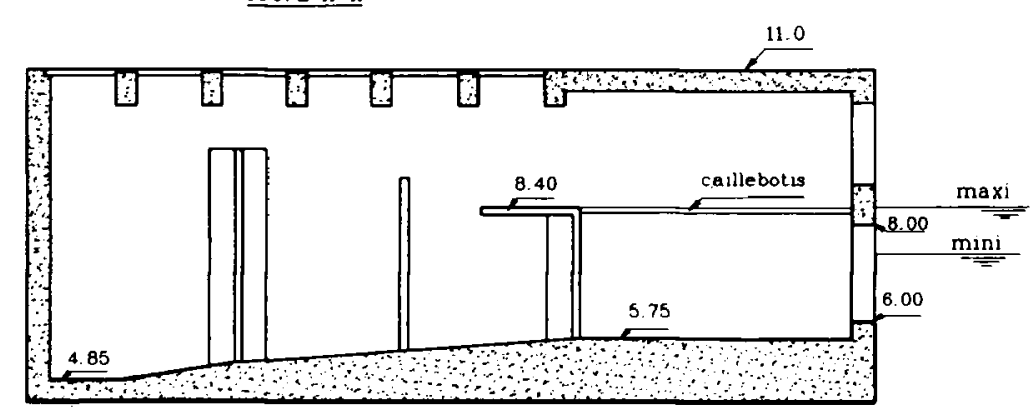

COUPE B-B

Figure 5 : Schémas de la passe à poissons estuarienne sur la Bresle.

Figure 5 : Views showing the estuarine fishway on the Bresle River. 


\section{BIBLIOGRAPHIE}

DANE B.G., 1983. A review and resolution of fish passage problems at culverts sites in British Columbia. Fisheries and Marine Serv., Vancouver, Rep. 810, 126 p.

DRYDEN R.L., STEIN J.M., 1975. Guidelines for the protection of the fish resources of the Northwest territories during highway construction and operation. Fisheries and Marine Serv., Tech. Rep. Series CEN/T-75-1, 32 p.

ENGEL P., 1974. Fish facilities for culverts of the McKenzie highway. Nat. Wat. Res. Inst., Burlington, Ontario, $33 \mathrm{p}$.

EUZENAT G., FOURNEL F., 1983. Etude préliminaire des conditions hydrauliques du passage mer/rivière des salmonidés migrateurs en Seine-Maritime. Problèmes du busage estuarien. Rapp. CSP, $8 \mathrm{p}$.

EUZENAT G., LARINIER M., 1990. Port de Pêche du Tréport, barrage-écluse. Dispositif de franchissement par les poissons migrateurs. Rapp. CSP, $65 \mathrm{p}$.

I.M.F.T., 1988a. Etude sur modèle réduit de l'écoulement dans les exutoires des rivières côtières de Seine-Maritime. Cas de l'Arques. Rapp. 436.2, 21 p.

I.M.F.T., 1988b. Etude sur modèle réduit de l'écoulement dans les exutoires des rivières côtières de Seine-Maritime. Ouvrage de rejet en mer. Rivière La Durdent. Rapp. 436.3, $38 \mathrm{p}$.

I.M.F.T., 1990. Port du Tréport. Etude sur modèles réduits d'une passe à poissons de l'évacuateur de crues du chenal de restitution. Rapp. 452.1, 65 p.

KATOPODIS C., 1984. Drainage design considerations for fish passage. Proc. of 3rd Annual Western Provincial Conf. Rationalization of Water and Soil Research and Management. Manitoba Water Resources Branch : 347-376.

KATOPODIS C., ROBINSON P.R., SUTHERLAND B.G., 1978. A study of model and prototype culvert baffling for fish passage. Fisheries and Marine Serv., Tech. Rep. $828,78 \mathrm{p}$.

KNIGHT D.W., MCDONALD J.A., 1979. Hydraulic resistance of artificial strip roughness. J. Hydr. Div., 105 (HY6) : 675-690.

MCKINLEY W.R., WEBB R.D., 1966. A proposed correction of migratory fish problems at box culverts. Fish. Res. Pap., 1(4) : 33-45.

RAJARATNAM N., KATOPODIS C., 1989. Hydraulics of culvert fishways II : slotted-weir culvert fishways. Can. J. Civ. Eng., 16 (3) : 375-383.

RAJARATNAM N., KATOPODIS C., 1990. Hydraulics of culvert fishways III : weir baffle culvert fishways. Can. J. Civ. Eng., 17 (4) : 558-568.

RAJARATNAM N., KATOPODIS C., LODEWYK S., 1988. Hydraulics of offset baffle culvert fishways. Can. J. Civ. Eng., 15 (6) : 1043-1051.

SAYRE W.W., ALBERTSON M.L., 1961. Roughness spacing in rigid open channels. J. Hydr. Div., 87 (HY3) : 121-150.

WATTS F.J., 1974. Design of culvert fishways. Dept. Civ. Eng., Univ. of Idaho, USA, 62 p. 




Photo 1 : Vue du débouché aval d'une buse illustrant les problèmes posés à la migration du poisson : chute à l'aval, vitesse élevée et tirant d'eau insuffisant dans l'ouvrage.

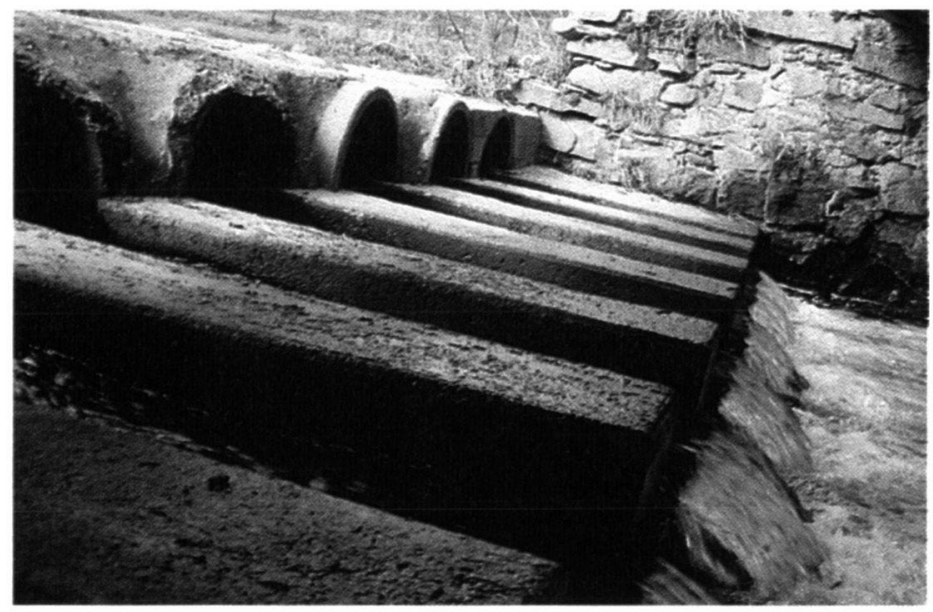

Photo 2 : Obstacle à la migration constitué par un pont-buse sur un ruisseau en Haute-Loire (cl. J.J. Demars).

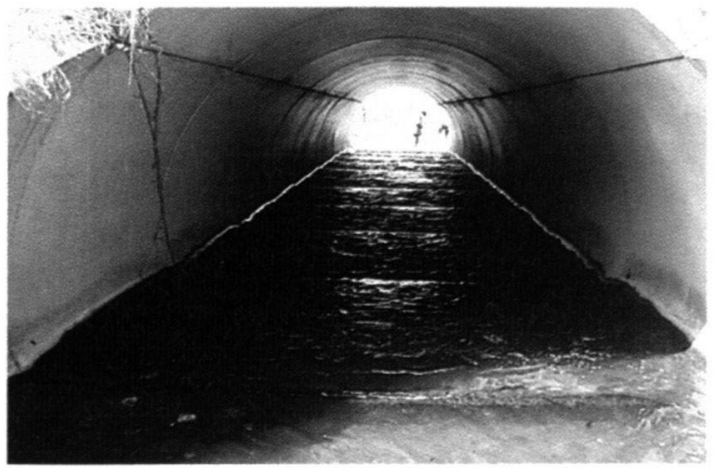

Photo 3 : Seuils rectangulaires dans une buse-arche.

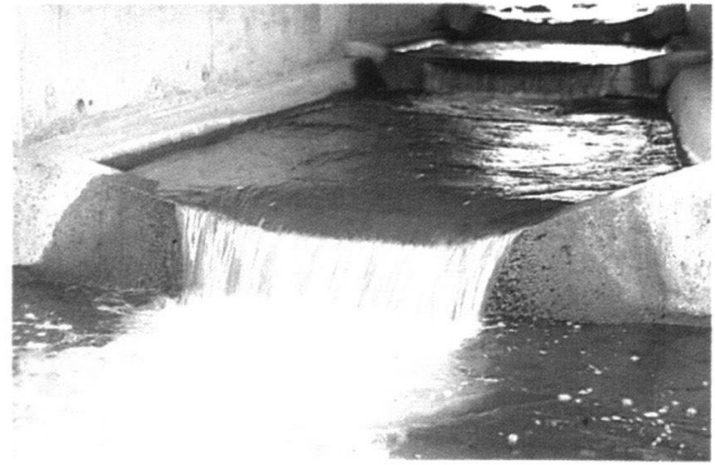

Photo 4 : Seuils triangulaires dans un pont-cadre. 
Bull. Fr. Pêche Piscic. (1992) 326-327 : 111-124 - $124-$

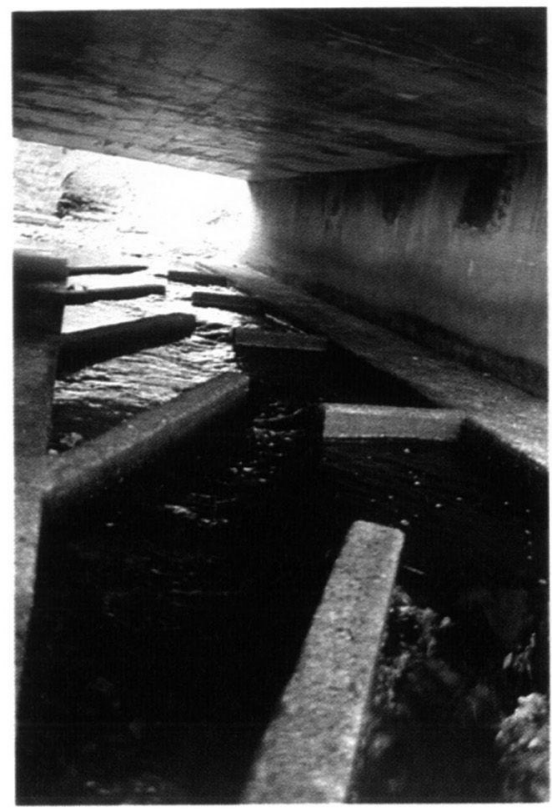

Photo 5 : Déflecteurs de type "offset".

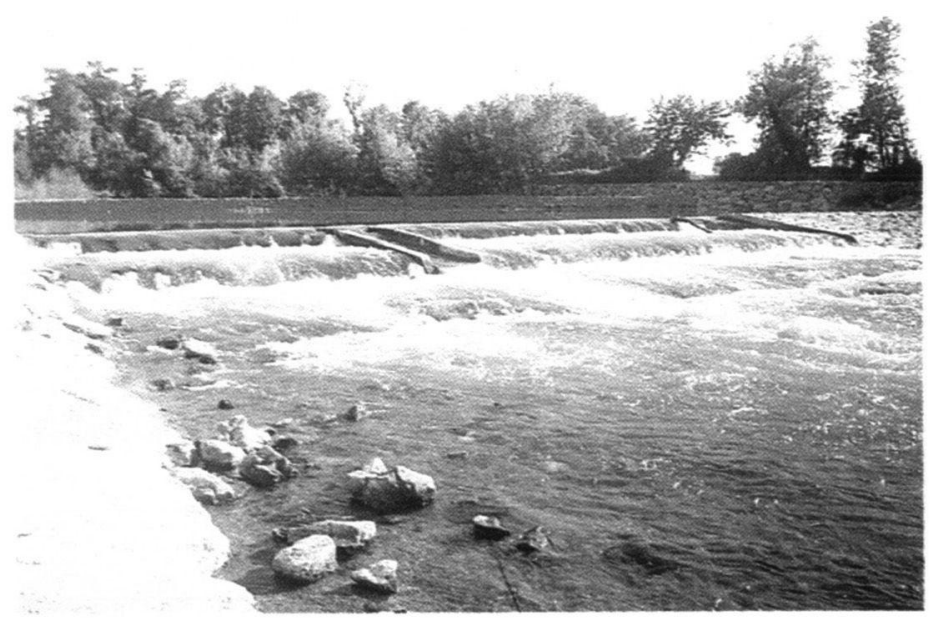

Photo 6 : Seuil en enrochements en gradins sur l'Adour (HautesPyrénées).

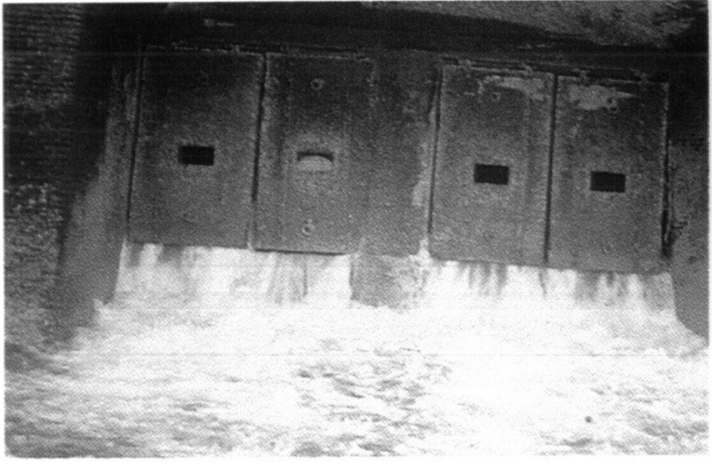

Photo 7 : Clapets à marée sur l'Arques à Dieppe (Seine-Maritime) (cl. J.L. Fagard).

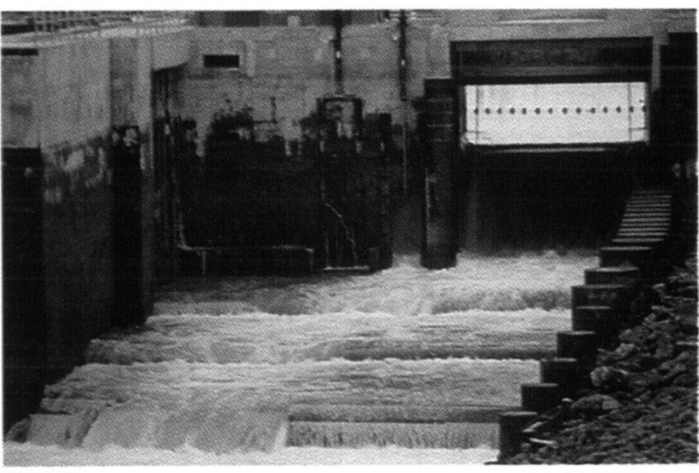

Photo 8 : Vue générale du barrage estuarien du Treport montrant les seuils permettant au poisson d'accèder à marée basse à l'entrée inférieure de la passe. A droite, le clapet de décharge (cl. G. Euzenat). 